\title{
MOLECULAR DYNAMICS SIMULATIONS OF LIQUID FLOW IN AND AROUND CARBON NANOTUBES
}

\author{
William D. Nicholls* \\ Department of Mechanical Engineering \\ University of Strathclyde \\ Glasgow G1 1XJ \\ United Kingdom \\ Email: william.nicholls@strath.ac.uk
}

\author{
Matthew K. Borg \\ Jason M. Reese \\ Department of Mechanical Engineering \\ University of Strathclyde \\ Glasgow G1 1XJ \\ United Kingdom \\ matthew.borg@strath.ac.uk \\ jason.reese@strath.ac.uk
}

\begin{abstract}
Using recently-developed fluid state controllers [1], we apply continuum fluid boundary conditions to molecular dynamics (MD) simulations of liquid argon flow past a carbon nanotube (CNT) and through a CNT membrane. Advantages of this method are that it: is not dependent on periodic boundary conditions; can accurately generate fluid transport without any geometrical constraints; and is capable of performing as an essential part of a hybrid continuum/atomistic technique. In our simulations, a pressure gradient is applied across a CNT membrane by controlling the densities of two reservoirs located either side of the membrane. Fluid velocity and density distributions are reported and compared to other published data where possible.
\end{abstract}

\section{INTRODUCTION}

Modelling complex fluid interactions with carbon nanotubes (CNTs) is vital for many nanoscale applications, such as developing CNT membranes for highly selective filtration or desalination [2]. Continuum models, such as the Navier-Stokes equations, are not capable of capturing flow phenomena around CNTs, and a molecular approach in these cases is always required $[3,4]$. The objective of this work is to investigate liquid

\footnotetext{
*Address all correspondence to this author.
}

flow in and around carbon nanotubes using molecular dynamics (MD).

One of the main problems in MD is the application of boundary conditions to generate fluid flow, in particular, pressure driven flow. Various methods exist in the literature to induce fluid transport in MD; one of the most common is the gravitational field method [5], which applies a gravitational force to all molecules in the system, the magnitude of which is much greater than the earth's gravitational pull. A drawback of this is that it is difficult to control applied pressure gradients and maximum flow velocities. Another method is the channel moving model [6], which creates a pressure gradient and hence fluid flow in a nanochannel by using two moving channel walls that drive the flow through viscous shear forces, towards a stationary wall at the end of the channel. While this method has been shown to produce constant pressure gradients, it is restricted in its application due to the geometrical constraints which it is reliant upon, i.e. parallel channel walls. The application of boundary conditions should be as general as possible and should be independent of the problem being simulated. The channel moving wall method is also not suitable for generating pressure gradients across membranes. In such a case an external force is applied to molecules in a region surrounding the membrane which, when coupled with periodic boundary conditions, generates a hydro- 
static pressure difference [7]. Huang et al. [8] proposed a method using two rigid self-adjusting plates located at the extremities of reservoirs either side of a nanoscale membrane. External forces are applied to the plates which correspond to the required constant pressures in each reservoir. This method has recently been developed in [9] to include flexible self adjusting plates to reduce the effect the rigid plates have on the molecular distributions near them.

In this work we use the new continuum boundary conditions of Borg et al. [1] to generate fluid transport. By introducing non-periodic boundary conditions (NPBCs) and controlling state quantities and fluxes in user-defined regions, we can impose continuum-like boundary conditions in the MD domain. An additional motive in the development of this method is that it enables MD simulations to be included as part of a hybrid continuum-molecular technique [10-13]; boundary conditions from the continuum solver are applied on the MD domain using these "controllers".

Two different problems are addressed in this paper: liquid argon flow past a CNT, and a CNT membrane separating two reservoirs of liquid argon at different densities creating a pressure gradient. Liquid argon was chosen as the working fluid due to its simplicity and is used as a test case.

\section{SIMULATION METHODOLOGY}

All our MD simulations were run in OpenFOAM, an opensource software which is available from [14]. The motion of molecules in an MD simulation is governed by Newton's second law. The equations of motion were integrated using the Verlet Leapfrog scheme. Interactions between all molecules, both liquid-liquid and liquid-solid, were represented by the LennardJones 12-6 potential:

$$
U_{i j}=4 \varepsilon_{i j}\left[\left(\frac{\sigma_{i j}}{\mathbf{r}_{i j}}\right)^{12}-\left(\frac{\sigma_{i j}}{\mathbf{r}_{i j}}\right)^{6}\right]
$$

where $U_{i j}$ is the potential energy, $r_{i j}$ is the distance between two interacting molecules $i$ and $j$, and $\varepsilon_{i j}$ and $\sigma_{i j}$ are characteristic energy and length scales. The parameters we used for the Lennard-Jones interactions are found in Table 1. A cut-off radius of $0.8 \mathrm{~nm}$ was used for both argon-argon and carbon-argon pairs. In all simulations the carbon atoms of the nanotubes were "frozen" to their original positions, making the nanotubes rigid, to reduce the computation time of the simulations. No interaction was included between carbon-carbon pairs. Continuum boundary conditions were applied on a cell by cell basis, cell values denoted below by the subscript $P$; the control process is fullyparallelised, when a domain decomposition method is used for parallel processing. The method of controlling the fluid towards a required state property or mass flow rate is based on a feedback
TABLE 1. LENNARD-JONES PARAMETERS.

\begin{tabular}{lcc}
\hline Interacting Pair $(i, j)$ & $\varepsilon_{i j}(\mathrm{~J})$ & $\sigma_{i j}(\mathrm{~m})$ \\
\hline Ar-Ar & $1.6567 \times 10^{-21}$ & $3.4 \times 10^{-10}$ \\
Ar-C & $1.9646 \times 10^{-21}$ & $3.573 \times 10^{-10}$ \\
\hline
\end{tabular}

loop algorithm: first, the property to be controlled is sampled over a given time period; second, the error between the measured value and the required value is calculated; and finally actions are taken to correct any error. Density is controlled by inserting or deleting molecules. For insertion, the USHER algorithm [15] is used which searches for a site within the potential energy landscape via a steepest descent scheme. A molecule is inserted if the potential energy is equal to the cell averaged potential energy. In this way, the algorithm ensures that no overlapping molecules occur nor does the local potential energy or temperature change after insertions/deletions. For deletion, the candidate molecule is chosen with its potential energy closest to the cell-averaged potential energy. The number of molecules which are required to be inserted or deleted over a specified time interval, $t \rightarrow t_{n}$, is calculated as follows:

$$
\Delta N_{P}\left(t \rightarrow t_{n}\right)=\mathrm{NINT}\left(\left[\rho_{P}^{\mathrm{req}}\left(t_{n}\right)-\rho_{P}^{\text {meas }}(t)\right] V_{P}\right)
$$

where NINT is a nearest integer function, as only whole molecules can either be inserted or deleted, $\rho_{P}^{\text {req }}\left(t_{n}\right)$ is the required density at the new time $t_{n}, \rho_{P}^{\text {meas }}(t)$ is the measured density at the old time $t$, and $V_{P}$ is the cell volume. The process of deleting or inserting molecules is performed over a number of control intervals which is defined by the user.

By adapting the USHER algorithm and only inserting/deleting molecules at a boundary of a domain, a mass flow rate can be applied and is defined by the following expression:

$$
\dot{m}_{f}=(\rho \mathbf{u})_{f} \cdot \mathbf{A}_{f},
$$

where $f$ is an arbitrary boundary face, $\mathbf{A}_{f}$ is the normal face area vector, and $(\rho \mathbf{u})_{f}$ uses face interpolated values for density and velocity. Using this method, the user has control over the applied mass flux at a boundary by specifying a value for $\dot{m}$. The outlet of a nano-channel can be modelled to adaptively control the flux of mass exiting the system in order to setup a pressure gradient across the system. This is done by selecting $\rho$ in Eqn. (3) to be the error between a target density (chosen smaller than that at the inlet) and a density measured in the domain. The convective velocity of the fluid may be controlled by applying an external force to the fluid molecules, in addition to the intermolecular 


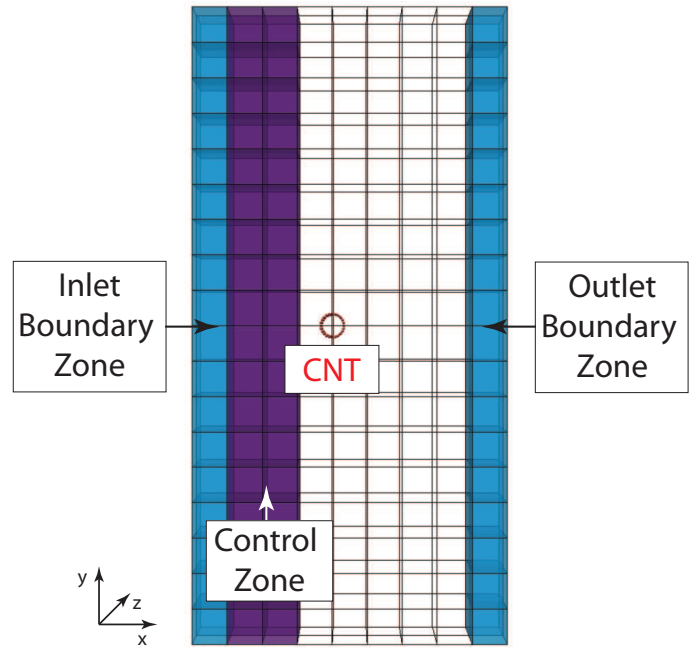

FIGURE 1. SIMULATION DOMAIN FOR CROSS-FLOW OF LIQUID ARGON OVER A CNT, WITH THE BOUNDARY AND CONTROL ZONES HIGHLIGHTED.

forces. The required velocity change over a given time period, $t \rightarrow t_{n}$, is calculated by using the following expression:

$$
\Delta \mathbf{u}_{P}\left(t \rightarrow t_{n}\right)=\lambda\left(\mathbf{u}_{P}^{\text {req }}\left(t_{n}\right)-\mathbf{u}_{P}^{\text {meas }}(t)\right),
$$

where $\mathbf{u}_{P}^{\mathrm{req}}\left(t_{n}\right)$ is the required velocity at the new time $t_{n}$ and $\mathbf{u}_{P}^{\text {meas }}$ is the measured velocity at the old time $t$. The imposition of the velocity difference, $\Delta \mathbf{u}_{P}\left(t \rightarrow t_{n}\right)$, is also divided over a number of control intervals defined by the user. A relaxation parameter $\lambda$ is used to control the rate at which the molecules are accelerated towards the target velocity. We found that a value of 1 gives generally good results. The external force required to obtain the appropriate velocity change can be calculated as follows:

$$
\mathbf{f}_{i}^{\text {ext }}=\frac{\Delta \mathbf{u}_{P}\left(t \rightarrow t_{n}\right)}{\Delta t_{m}} m_{i}
$$

where $\Delta t_{m}$ is the simulation time step size and $m_{i}$ is the mass of molecule $i$. These controllers are described in more detail in [1].

\section{Liquid Argon Flow Past a CNT}

First we validate the use of our velocity controller by repeating a simulation of the cross-flow of liquid argon past a CNT, originally performed by Tang and Advani [16]. The original simulation initialised fluid flow by superimposing the required velocity, $158 \mathrm{~m} / \mathrm{s}$, on all argon atoms in a control region upstream of the CNT, creating uniform flow. Instead, we controlled velocity by exerting an additional force on the molecules in the control region, which is calculated using Eqns. (4) and (5). The domain size was $30.56 \times 25.47 \times 2.23 \mathrm{~nm}$ in the $x, y$ and $z$ directions, respectively, and periodic boundary conditions were applied in every direction, see Fig. 1 . The simulation time was $8.6 \times 10^{-10}$ $\mathrm{s}$ with a time step of $2.15 \times 10^{-15} \mathrm{~s}$. The fluid density was 1342 $\mathrm{kg} / \mathrm{m}^{3}$. A Berendsen thermostat was used in our simulation to remove the excess heat (which is created due to the application of the additional force) and maintain the system at $95 \mathrm{~K}$. The average velocity profile is measured across the centre of the nanotube using a binning method, described in detail in [16], between $4.3 \times 10^{-10} \mathrm{~s}$ and $8.6 \times 10^{-10} \mathrm{~s}$, which is the equivalent of 200000 time steps.

Since we are not interested in what happens downstream of the nanotube in this particular problem, the domain size can be reduced to decrease the computation time. However, continuing to use periodic conditions in this situation is not possible, as the depletion zone which is created in the "wake" of the CNT is then reintroduced at the inlet of the domain, significantly affecting the results. In order to reduce the size of the domain, we must therefore use non-periodic boundary conditions. The domain size was reduced in the $x$ direction, but not in the $y$ and $z$ directions in order to compare results fairly. The periodic boundaries in the $x$ direction were replaced by non-periodic boundaries with two boundary zones at the inlet and outlet of the domain, as shown in Fig. 1. The control zone remained unchanged from the initial validation; controlling velocity at $158 \mathrm{~m} / \mathrm{s}$ and temperature at $95 \mathrm{~K}$. The inlet and outlet boundary zones were composed of a stochastic thermal wall at the termination of the domain, and a mass flux boundary which inserts and deletes molecules, calculated using Eqn. (3), only in the boundary zones. Molecules that collide with the stochastic thermal wall are reflected back into the boundary zone with their velocities resampled from a Maxwell-Boltzmann distribution at a prescribed boundary-face temperature, $95 \mathrm{~K}$, and mean velocity, $158 \mathrm{~m} / \mathrm{s}$. The density of argon and the time characteristics of the simulation otherwise remained unchanged.

No additional force has been included in the boundary zone to account for the missing interactions with molecules which would be present beyond the boundary, as previously performed by Borg et al. [1]. This should not greatly affect the results as the regions of interest are located far enough away from the boundary zones.

\section{Liquid Argon Flow Through a CNT Membrane}

The second MD case consisted of flow through a CNT embedded in a solid substrate. Two semi-infinite reservoirs on either side of the CNT membrane were set at different pressures as shown in Fig. 2. The domain geometry was $24 \times 7 \times 7 \mathrm{~nm}$ in the $x, y$, and $z$ directions, respectively, which closely matches a previous simulation performed by Huang et al. [8]. The pressure in each reservoir was indirectly controlled by particle in- 


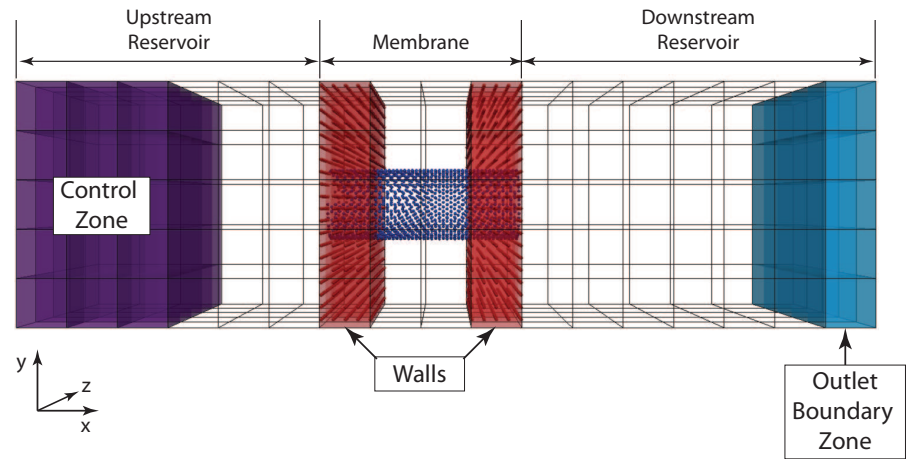

FIGURE 2. SIDE-VIEW OF SIMULATION DOMAIN FOR FLOW OF LIQUID ARGON THROUGH A CNT MEMBRANE, SHOWING THE CNT MEMBRANE, AND BOUNDARY AND CONTROL ZONES.

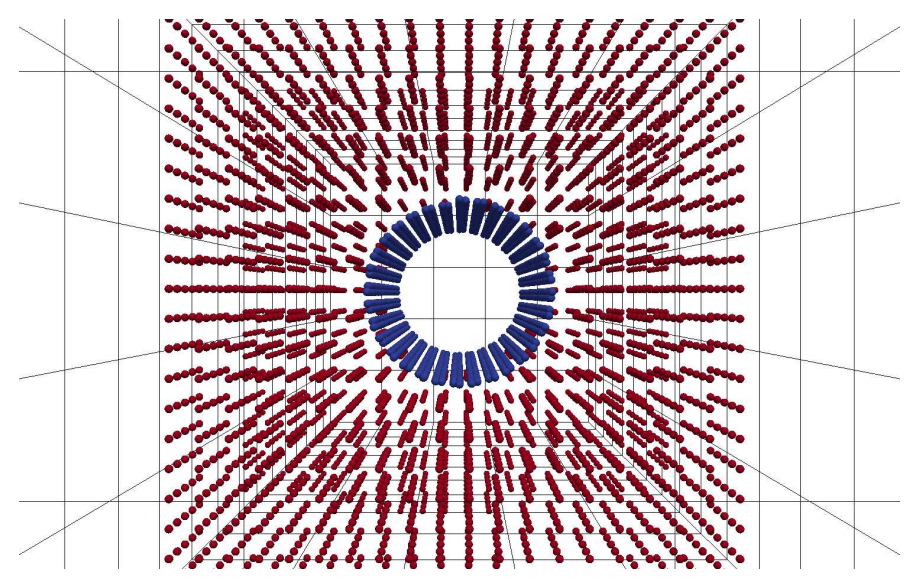

FIGURE 3. END-ON VIEW OF THE CNT EMBEDDED IN A SOLID SUBSTRATE.

sertion and deletion only. In order to investigate fluid transport due to a pressure gradient, Berendsen thermostats were applied to each reservoir to maintain a constant temperature of $133 \mathrm{~K}$ and eliminate the contribution of any temperature gradients to the fluid transport. A $(16,16) \mathrm{CNT}$ with a radius of $1.085 \mathrm{~nm}$ and length of $6 \mathrm{~nm}$ was used. The two membrane surfaces which surround the inlet and outlet of the CNT were constructed of the same molecules as the fluid, but frozen to their original positions and do not interact with one another, see Fig. 3. The density of the solid substrate was $1500 \mathrm{~kg} / \mathrm{m}^{3}$. The reservoir upstream of the CNT was controlled to be at a density of $1312 \mathrm{~kg} / \mathrm{m}^{3}$ and the downstream reservoir $653 \mathrm{~kg} / \mathrm{m}^{3}$. The average number of argon molecules in the system was $\approx 18000$, although this number varied due to the insertion and deletion of molecules. The upstream reservoir's density was controlled using a density controller, Eqn. (2), in the control zone highlighted in Fig. 2. The

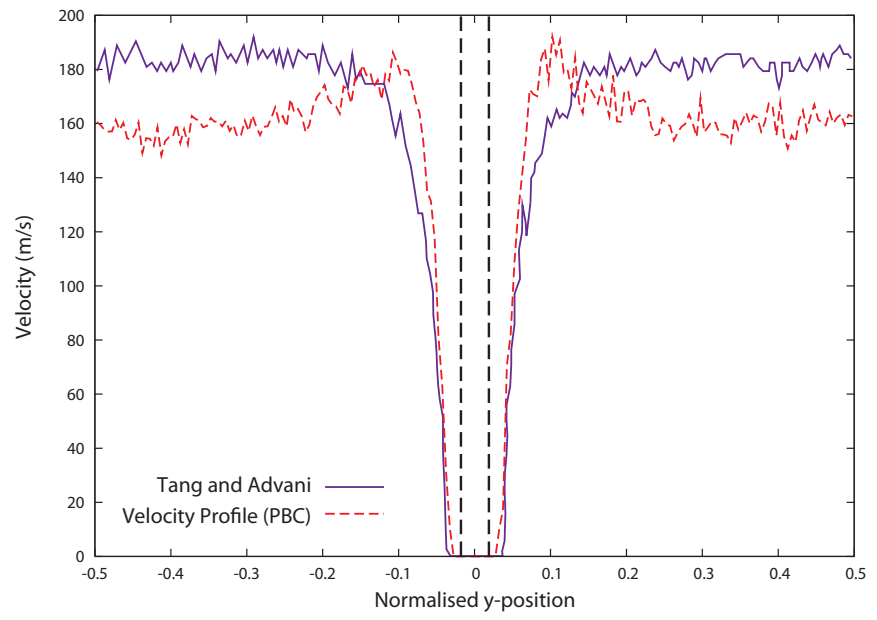

FIGURE 4. STREAMING VELOCITY PROFILES FOR FLOW PAST A CNT IN A FULLY PERIODIC SYSTEM; THE VERTICAL DASHED LINES INDICATE THE POSITION OF THE CNT.

insertion and deletion of molecules was located far away from the CNT membrane so as not to disturb important entrance effects which play a vital role in fluid transport through nanoscale membranes [9]. A time step of $1 \times 10^{-15} \mathrm{~s}$ was chosen to ensure that no molecules would overlap after any time step.

Two simulations were performed to investigate the effect of different outlet boundary representations. In the first simulation, density was controlled in the downstream reservoir (4.2 $\mathrm{nm}$ thick), similar to the upstream case. In the second simulation, a pressure gradient was enforced by using the outlet mass flux model described previously. The density distribution downstream of the CNT membrane may not be uniform and it is important to capture such behaviour and not affect this by particle insertion/deletion.

\section{RESULTS AND DISCUSSION Liquid Argon Flow Past a CNT}

The streaming velocity profile obtained for the flow of liquid argon past a CNT in a fully periodic domain is presented in Fig. 4, where it is compared to published data by Tang and Advani [16].

It is clear from Fig. 4 that Tang and Advani have not created the correct freestream velocity by their method of superimposing the required velocity on argon molecules upstream of the CNT. The required fluid velocity was $158 \mathrm{~m} / \mathrm{s}$, their measured value is over $180 \mathrm{~m} / \mathrm{s}$. Using our velocity controller we have successfully created a free stream velocity of $158 \mathrm{~m} / \mathrm{s}$, shown in Fig. 4 .

The liquid argon can be seen accelerating around the nanotube reaching a maximum velocity of $\sim 180 \mathrm{~m} / \mathrm{s}$; no such behaviour is present in the profile produced by Tang and Advani. 


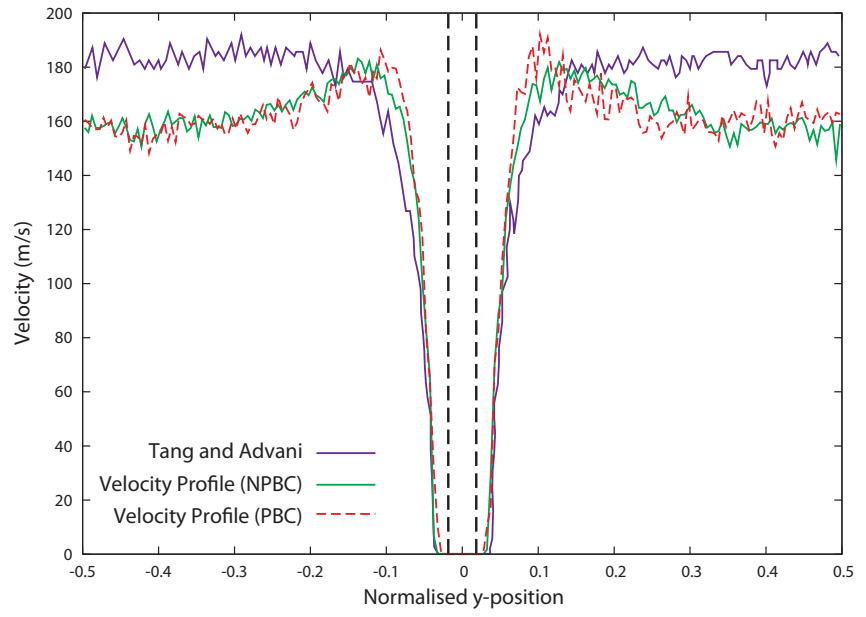

FIGURE 5. STREAMING VELOCITY PROFILES FOR FLOW PAST A CNT IN A FULLY PERIODIC SYSTEM (PBC), AND A SYSTEM WITH NON-PERIODIC BOUNDARY CONDITIONS (NPBC) IN ONE DIRECTION.

It has also been suggested that higher flow rates produce greater values for slip velocity, which is attributed to larger velocity gradients close to the CNT [16]. However it may be observed from Fig. 4 that we have a greater velocity gradient close to the nanotube with a lower, more accurate, fluid velocity. The two velocity profiles converge close to the CNT surface in a density depletion zone created by the repulsion force of the nanotube.

By introducing NPBCs and mass flux boundary conditions we were able to reduce the size of the simulation domain and decrease the computation time of the problem. Using the same binning method as the fully periodic case, we obtained the velocity profile across the CNT and have compared this to results by Tang and Advani and the previous case in Fig. 5. The velocity profiles for the PBC and NPBC cases are in very good agreement. It is clear from Fig. 5 that the reduction of the domain size and introduction of NPBCs has had no effect on the velocity distribution around the CNT; but the simulation now runs approximately 1.7 times faster than the fully periodic case. The temperature of the system remained at a constant value of $98 \mathrm{~K}$, which is slightly higher than the required value of $95 \mathrm{~K}$. This error may be reduced by altering the control parameters e.g. the number of time steps between control sequences.

\section{Liquid Argon Flow Through a CNT Membrane}

In order to obtain density and velocity profiles in the CNT radial direction, a cylindrical binning method was employed. A cylinder of radius $0.5 \mathrm{~nm}$ was placed at the centre of the domain, spanning the full domain height in the $y$ direction. This cylindrical region was divided into 32 bins, with 10 lying inside of the CNT. The simulations were allowed to run for 200000 time steps before averaging properties to allow the system to reach steadystate conditions. Results were obtained by averaging over one million time steps.

Density profiles obtained from both simulations are shown in Fig. 6. The profiles each possess four distinct peaks, with the largest adjacent to the CNT surface, which indicates the formation of liquid layers. This behavior has previously been observed in Poiseuille flow simulations of confined liquids in nanoscale channels and pores $[8,17,18]$. The fluid layering intensity is directly influenced by the wall-fluid interaction [18] and parameters chosen to represent such interactions [17]. Figure 6 clearly shows for both simulations that the fluid density close to the CNT walls is zero, which is in agreement with results by Travis and Gubbins [18], but results by Huang et al. [8] show no evidence of this density depletion adjacent to the wall. The results in Fig. 6 show that the outlet representation does not alter the shape of either density profile. However a larger fluid density is present at the centre of the CNT for the case with the mass flux control at the outlet. This difference may be explained by examining the density distributions along the full domain in the axial direction of the CNT, as shown in Fig. 7.

Upstream conditions are controlled in the same manner: fixed density and temperature. As a result, the density distributions are almost identical, as expected. The fluid densities for both cases are fairly constant along the length of the CNT with the mass flux outlet case being slightly higher, as previously shown in Fig. 6. The density in the downstream reservoir for the fixed density outlet case drops sharply at the outlet of the CNT before recovering to the controlled value towards the end of the reservoir. The density distribution for the mass flux outlet case is an almost constant density from the outlet of the CNT to a distance of $\sim 3 \mathrm{~nm}$ into the downstream reservoir, before dropping sharply towards the reservoir boundary. The gradients of the sharp changes in density are almost identical, which implies that if the downstream reservoir were to be made longer, i.e. moving the controlling region further away from the membrane, then the uniform density distribution may be extended over a larger portion of the reservoir.

Streaming velocity profiles inside the CNT from both simulations are shown in Fig. 8. The obtained velocity profiles are not quadratic in form, which is what would have been predicted by continuum fluid theory for Poiseuille flow conditions, however they are very similar in shape to profiles obtained from similar simulations [18]. It is clear from Fig. 8 that the outlet representations have a significant effect on the velocity distributions inside the CNT, with the maximum velocity for the fixed density outlet case being over twice as large as that for the mass flux outlet case. This is due to the different density distributions present in each downstream reservoir, as previously discussed. The immediate drop in density for the fixed density outlet case is inducing a higher flow rate along the CNT.

The small fluctuations present in both measured velocity 


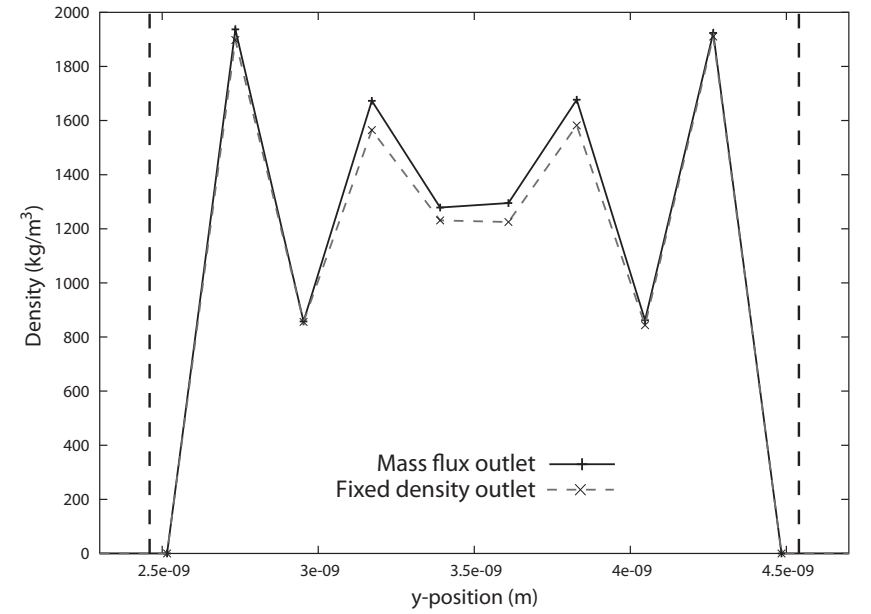

FIGURE 6. EFFECT OF OUTLET REPRESENTATION ON MASS DENSITY PROFILES INSIDE CNT; THE VERTICAL DASHED LINES INDICATE THE INTERNAL SURFACES OF THE CNT.

profiles are a result of the previously described binning method. Due to the layered fluid structure inside the CNT; the bin size becomes more influential. Using too fine a bin resolution will result in some bins having no molecules inside them, due to the layer structure, and will create more fluctuations in the measured profile. Using too coarse a resolution will not capture accurately the form of the profile. An alternative measurement technique is the method of planes [19], which is not as susceptible to random error due to the variation in measurement resolution; it was previously used to calculate density and velocity profiles in nanoscale channels [18].

\section{CONCLUSIONS}

Using recently developed controllers [1] we have applied continuum fluid boundary conditions in MD to simulate liquid argon flow past a CNT and through a CNT membrane. Advantages of our method are that it: is not dependent upon periodic boundary conditions; can accurately generate fluid transport without any geometrical constraints; and is capable of performing as an essential part of a hybrid continuum-atomistic technique. Results obtained using MD in OpenFOAM for the CNT membrane case show that velocity and density profiles inside the CNT membrane are similar in form to previous published results $[8,17,18]$, with the density distribution being oscillatory and the velocity distribution having a quasiparabolic profile. More work is required to address the issue of non-uniform density distributions in the downstream reservoir. The inclusion of the missing boundary forces will also be subject of future work. In general, our results show that our method of creating fluid transport at the nanoscale produces more accurate velocity profiles for flow past a CNT

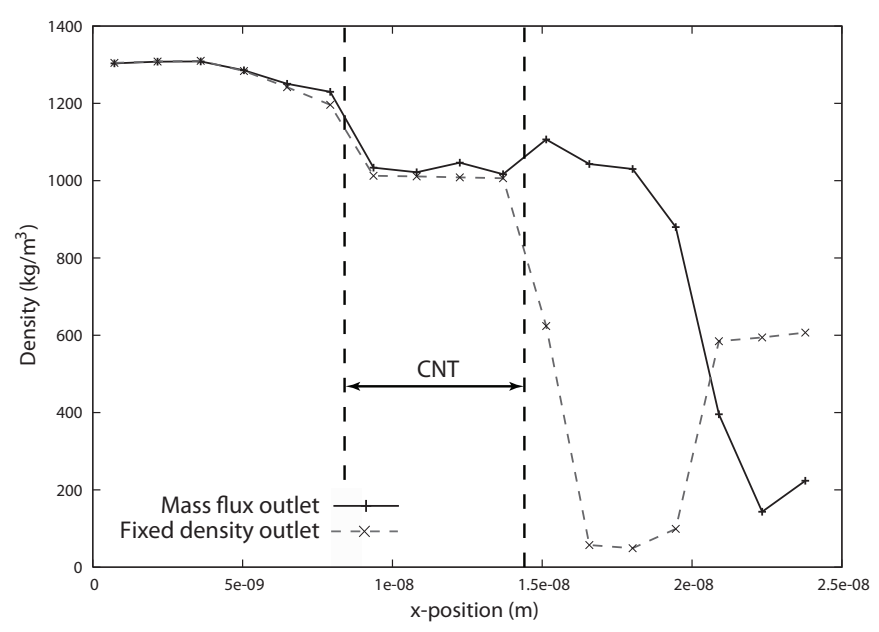

FIGURE 7. MASS DENSITY DISTRIBUTIONS THROUGH THE LENGTH OF THE DOMAIN UNDER DIFFERENT OUTLET CONDITIONS; THE VERTICAL DASHED LINES INDICATE THE INLET AND OUTLET POSITIONS OF THE CNT MEMBRANE.

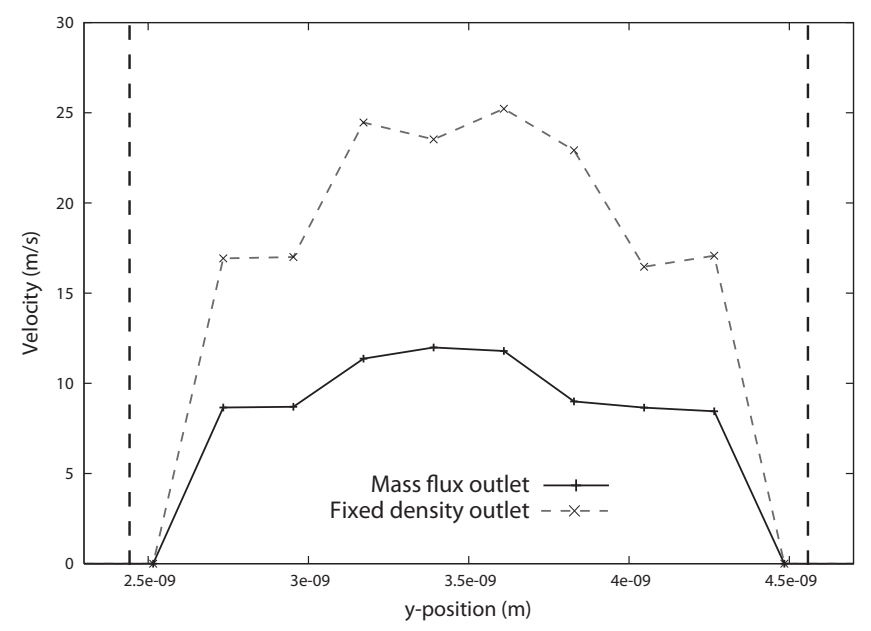

FIGURE 8. EFFECT OF OUTLET REPRESENTATION ON VELOCITY PROFILES INSIDE CNT; THE VERTICAL DASHED LINES INDICATE THE INTERNAL SURFACES OF THE CNT.

compared to previous methods.

This work is currently being extended to enable the control of water molecules using an adapted USHER algorithm for polar polyatomic molecules [20]. Long range electrostatic interactions can be calculated using a two-dimensional particle mesh Ewald summation $[8,21]$. 


\section{ACKNOWLEDGMENT}

Financial support from the EPSRC and the Institution of Mechanical Engineers is gratefully acknowledged. The authors thank Richard Martin of the University of Strathclyde for useful discussions.

\section{REFERENCES}

[1] Borg, M. K., Macpherson, G. B., and Reese, J. M. "Controllers for imposing continuum-to-molecular boundary conditions in arbitrary fluid flow geometries". In press, Molecular Simulation.

[2] Corry, B., 2008. "Designing carbon nanotube membranes for efficient water desalination". Journal of Physical Chemistry B, 112(5), pp. 1427-1434.

[3] Tuzun, R. E., Noid, D. W., Sumpter, B. G., and Merkle, R. C., 1996. "Dynamics of fluid flow inside carbon nanotubes". Nanotechnology, 7(3), pp. 241-246.

[4] Travis, K. P., Todd, B. D., and Evans, D. J., 1997. "Departure from navier-stokes hydrodynamics in confined liquids". Phys. Rev. E, 55(4), pp. 4288-4295.

[5] Koplik, J., Banavar, J. R., and Willemsen, J. F., 1988. "Molecular dynamics of poiseuille flow and moving contact lines". Physical Review Letters, 60(13), pp. 1282-1285.

[6] Zhang, Z., Zhang, H., and Ye, H., 2009. "Pressure-driven flow in parallel-plate nanochannels". Applied Physics Letters, 95(15), pp. 1-3.

[7] Zhu, F., Tajkhorshid, E., and Schulten, K., 2002. "Pressureinduced water transport in membrane channels studied by molecular dynamics". Biophysical Journal, 83(1), pp. 154 $-160$.

[8] Huang, C., Nandakumar, K., Choi, P. Y. K., and Kostiuk, L. W., 2006. "Molecular dynamics simulation of a pressure-driven liquid transport process in a cylindrical nanopore using two self-adjusting plates". Journal of Chemical Physics, 124(23), pp. 1-8.

[9] Huang, C., Choi, P. Y. K., Nandakumar, K., and Kostiuk, L. W., 2008. "Investigation of entrance and exit effects on liquid transport through a cylindrical nanopore". Physical Chemistry Chemical Physics (Incorporating Faraday Transactions), 10, pp. 186-192.

[10] O'Connell, S., and Thompson, P., 1995. "Molecular dynamics-continuum hybrid computations: a tool for studying complex fluid flows". Physical Review E, 52(6), pp. R5792-R5795.

[11] Delgado-Buscalioni, R., and Coveney, P., 2003. "Continuum-particle hybrid coupling for mass, momentum, and energy transfers in unsteady fluid flow". Physical Review E, 67(4), pp. 1-13.

[12] Werder, T., Walther, J., and Koumoutsakos, P., 2005. "Нybrid atomistic-continuum method for the simulation of dense fluid flows". Journal of Computational Physics, 205(1), pp. 373 - 390.

[13] Flekkøy, E. G., Delgado-Buscalioni, R., and Coveney, P. V., 2005. "Flux boundary conditions in particle simulations". Physical Review E, 72(2), pp. 1-9.

[14] OpenCFD. http://www.opencfd.co.uk/openfoam/.

[15] Delgado-Buscalioni, R., and Coveney, P. V., 2003. "Usher: An algorithm for particle insertion in dense fluids". Journal of Chemical Physics, 119(2), pp. 978-987.

[16] Tang, W., and Advani, S. G., 2006. "Drag on a nanotube in uniform liquid argon flow". Journal of Chemical Physics, 125(17), pp. 1-8.

[17] Liu, C., and Li, Z., 2009. "Flow regimes and parameter dependence in nanochannel flows". Phys. Rev. E, 80(3), pp. 1-5.

[18] Travis, K. P., and Gubbins, K. E., 2000. "Poiseuille flow of lennard-jones fluids in narrow slit pores". Journal of Chemical Physics, 112(4), pp. 1984-1994.

[19] Daivis, P. J., Travis, K. P., and Todd, B. D., 1996. "A technique for the calculation of mass, energy, and momentum densities at planes in molecular dynamics simulations". The Journal of Chemical Physics, 104(23), pp. 9651-9653.

[20] Fabritiis, G. D., Delgado-Buscalioni, R., and Coveney, P. V., 2004. "Energy controlled insertion of polar molecules in dense fluids". Journal of Chemical Physics, 121(24), pp. 12139-12142.

[21] Kawata, M., and Nagashima, U., 2001. "Particle mesh ewald method for three-dimensional systems with twodimensional periodicity". Chemical Physics Letters, 340(1-2), pp. $165-172$. 